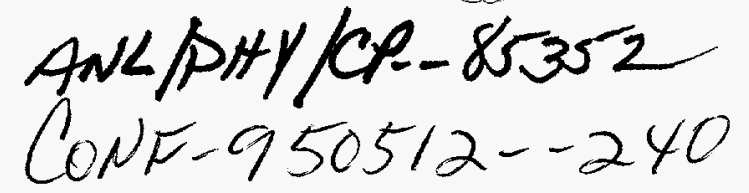

\title{
LONGITUDINAL EMITTANCE OSCILLATION IN A SUPERCONDUCTING DRIFT TUBE LINAC
}

\author{
J.W. Kim and K.W. Shepard, Argonne National Laboratory, Argonne, IL 60439 USA
}

In drift tube linacs a beam energy spread results from the finite beam size. Radial variation of the axial accelerating field induces a beam energy spread, which, in general, will accumulate as the beam passes through successive drift tubes. This paper shows that under some conditions of periodic transverse focusing and longitudinal phase focusing, the correlation between the longitudinal and transverse motion can be used to correct the energy spread. The process of achieving such a correction is first described in a simplified situation, and then demonstrated for a particular tuning using a ray-tracing program which models a low velocity and low charge state linac designed for radioactive ion beams.

\section{INTRODUCTION}

The design of an ISOL-type radioactive beam facility utilizing the present ATLAS accelerator as a secondary beam accelerator is described in several papers at this conference [1] [2]. One requirement for such a project is a low charge state injector linac for the ATLAS superconducting linac. A key issue with such an injector linac is to maintain small longitudinal emittance while maximizing transverse acceptance. These two requirements tend to conflict since acceleration of a finite size beam through a drift tube linac increases the beam energy spread because of radial variation of the accelerating field. The variation is quadratic in the lowest order, causing longitudinal beam quality to deteriorate rapidly with increasing beam radius. The beam energy spread is inversely proportional to the wavelength of the slow wave in the drift tube structure, thus becoming worst at low particle velocities.

The longitudinal emittance increase could in principle accumulate throughout the acceleration process. However, by proper matching of longitudinal phase focusing to the periodic transverse focusing structure, emittance growth can be limited by using the correlation between longitudinal and transverse phase spaces. This process is clearly manifested in numerical ray-tracing studies performed in the design of the low charge state ( $q / A \geq 1 / 66$ ), low velocity $(\beta \geq 0.004)$ injector linac described in reference [2]. In what follows, first a simplified version of the correction mechanism is described, and then numerical ray-tracing results are presented.

\section{A MECHANISM OF LONGITUDINAL EMITTANCE CORRECTION}

Figure 1 shows schematically a linac in which transverse focusing elements alternate with short drift tube accelerat- ing structures, resonant cavities, which are operated in a phase focusing mode. The cell length is $l_{\text {cell }}\left(=l_{\text {res }}+l_{\text {drift }}\right)$, and the stability condition of transverse motion in such a structure is that $f / l_{\text {cell }} \geq 1 / 4$, where $f$ is the focal length [3].

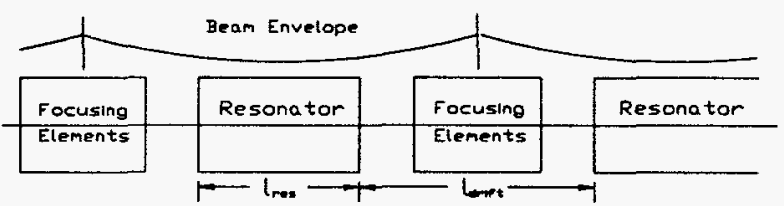

Figure 1: A periodic focusing structure in a drift tube linac along with a matched beam envelope.

In considering the behavior of a beam through the structure, we make several simplifying assumptions. First, the transverse focal length is a half of the cell length so that the phase advance of the transverse motion is $90^{\circ}$, which implies that all particles have the same radial displacements every other cell. Second, we consider only the energy spread induced by the first and third resonators (i.e. assume the second resonator has no radial variation in accelerating field).

A correction mechanism is depicted in Figure 2 for a three cell structure. The upper graphs show the longitudinal phase space, and the lower graphs display the correlation, showing energy spread versus radial displacement. Figure 2 (a) shows a beam energy spread induced at the first resonator. The longitudinal emittance increase is indicated by shading. On going through the second resonator, shown in Figure 2 (b), the matched phase focusing action of the resonator effectively inverts the energy spread. On arrival at the third resonator, the particles have advanced $180^{\circ}$ in transverse motion so that the particles repeat the displacement they had in the first resonator, but the energy spread is inverted. The beam bunch is thus effectively 'pre-compensated' for the radial variation in energy gain about to be experienced in traversing the third resonator. The beam energy spread is then perfectly corrected at the third resonator as shown in Figure 2 (c).

We can examine the action of the second resonator more quantitatively as follows. We define $\mathrm{U}\left(\phi_{o p}\right)$ to be a slope of the energy gain versus operating phase angle at $\phi_{o p}$. An approximate condition for matched phase focusing for ions of velocity $\beta$ is then

$$
U\left(\phi_{o p}\right) \simeq \frac{A m c^{3} \beta^{3}}{q \omega l_{c e l l}},
$$




\section{DISCLAIMER}

This report was prepared as an account of work sponsored by an agency of the United States Government. Neither the United States Government nor any agency thereof, nor any of their employees, makes any warranty, express or implied, or assumes any legal liability or responsibility for the accuracy, completeness, or usefulness of any information, apparatus, product, or process disclosed, or represents that its use would not infringe privately owned rights. Reference herein to any specific commercial product, process, or service by trade name, trademark, manufacturer, or otherwise does not necessarily constitute or imply its endorsement, recommendation, or favoring by the United States Government or any agency thereof. The views and opinions of authors expressed herein do not necessarily state or reflect those of the United States Government or any agency thereof. 


\section{DISCLAIMER}

Portions of this document may be illegible in electronic image products. Images are produced from the best available original document. 

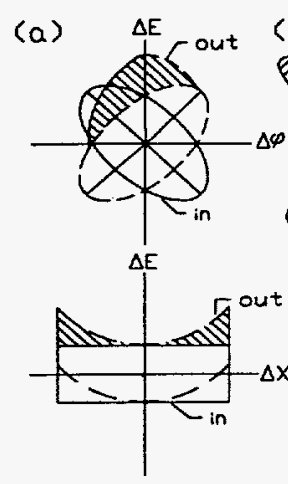

lst cell

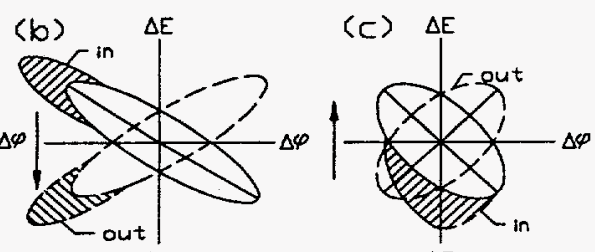
$\Delta E$ E

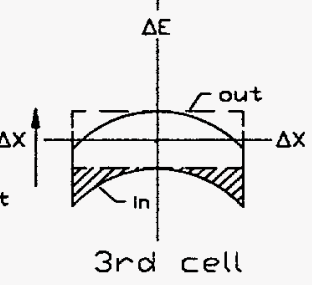

Figure 2: A simplified model for demonstrating the mechanism for correcting the energy spread resulting from radial variation of the accelerating field. The solid line indicates the phase space at the entrance of the resonator, and the dashed line at the exit. See the text for details.

where $\omega$ is the angular radio frequency. This condition can be satisfied by adjusting the operating phase angle $\phi_{\text {op }}$. The above relation has two implications of interest:

1) in going to low particle velocities, we must reduce the drift length, or $U\left(\phi_{o p}\right)$ by shifting the operating phase angle closer to the maximum energy gain phase (e.g. see Figure 3 in the next section),

2 ) in going to smaller $\mathrm{q} / \mathrm{A}$, however, we require stronger phase focusing for matching, which requires increasing the operating phase angle,

In the present application, 2) above is beneficial in allowing the operating phase angle to be sufficiently large to provide good linearity in the phase focusing. These effects have been observed with the ray-tracing calculations in a low charge state linac described in the next section.

When the phase focusing is not well matched to the linac structure, emittance correction is weaker, and correction period spreads out over more cells.

\section{NUMERICAL SIMULATIONS}

The above corrective process clearly appears in numerical simulations of a proposed low energy injector linac for ATLAS [2]. Phase space motion in such a linac was calculated using a ray-tracing program developed at ATLAS. The design particle is ${ }^{132} \mathrm{Sn}^{2+}$, and the initial velocity $(\beta)$ is 0.008 . The focusing element is a quadrupole triplet, perfect quadrupole fields with hard edges being assumed in the program. A realistic quadrupole design [4] can give a phase advance of $60^{\circ}$ with $f=l_{\text {cell }}$. The drift tube structures modeled in the program are the quarter wave superconducting resonators used at the low energy end of PII linac at ATLAS [5]. The linac parameters which are input to the program are listed in Table.1. The operating
Table 1: Input parameters for the ray-tracing program

\begin{tabular}{|c|c|}
\hline Resonator & $48.5 \mathrm{MHz}, 4$ gap \\
\hline$\beta_{\text {in }}, \phi_{0}$ & $0.008,-20^{\circ}$ \\
\hline Quadrupole strength & $350 \mathrm{~T} / \mathrm{m}$ \\
\hline Triplet length $(\mathrm{cm})$ & $(\mathrm{Q} 1, \mathrm{Q} 2, \mathrm{Q} 3)=(4,7,4)$ \\
\hline$l_{\text {res }}, l_{\text {drift }}$ & $14 \mathrm{~cm}, 29 \mathrm{~cm}$ \\
\hline$\beta_{\text {out }}$, after 7 resonators & 0.013 \\
\hline
\end{tabular}

phase and energy gain relationship of the I1-type resonator is shown in Figure 3 for ${ }^{132} \mathrm{Sn}^{2+}$ at a mean energy gain of $4.5 \mathrm{MeV} / \mathrm{m}$. The slope $\mathrm{U}\left(\phi_{o p}\right)$ is roughly $0.2 \mathrm{MeV} / \mathrm{rad} / \mathrm{q}$ at an operating phase of $-20^{\circ}$, which satisfies the condition (1) for $\beta$ near 0.009 .

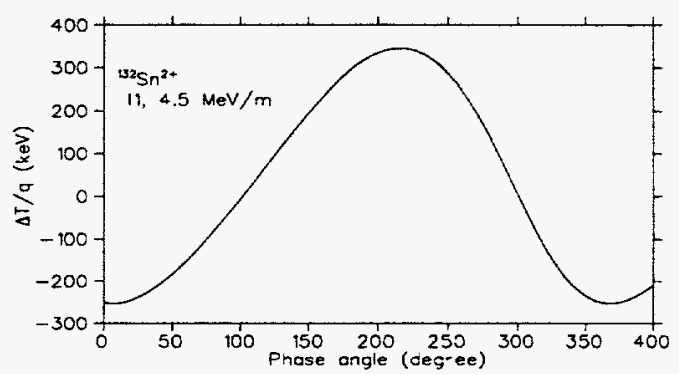

Figure 3: The operating phase angle-energy gain curve of $\mathrm{I} 1$ resonator for ${ }^{132} \mathrm{Sn}^{2+}$ at an average energy gain of $4.5 \mathrm{MeV} / \mathrm{m}$

Figure 4 shows the longitudinal phase space and Figure 5 the coupled phase space at the entrance and exit of the first, second, and fifth resonators. The normalized transverse emittance is $0.6 \pi \mathrm{mm} \cdot \mathrm{mrad}$, and the longitudinal emittance is $5 \pi \mathrm{keV} \cdot \mathrm{nsec}$. In (a) of Figure 4 and 5 the beam energy spread at the exit of the first resonator is clearly shown. In (b) of Figure 4 and 5 the process of inversion of the energy spread through phase focusing is manifested. In (c) of Figure 4 and 5 we clearly see that the energy spread is cancelled by the effect of the radial variation in energy gain through the fifth resonator.

The longitudinal emittance on the first seven resonators of the injector linac is displayed in Figure 6. The emittance increase and correction extends over the first five resonators. In fact the emittance correction process continues throughout the linac $(0.008 \geq \beta \geq 0.03)$, and longitudinal emittance appears to be oscillatory [2]. The oscillation amplitude becomes larger for a beam having larger transverse emittance. 

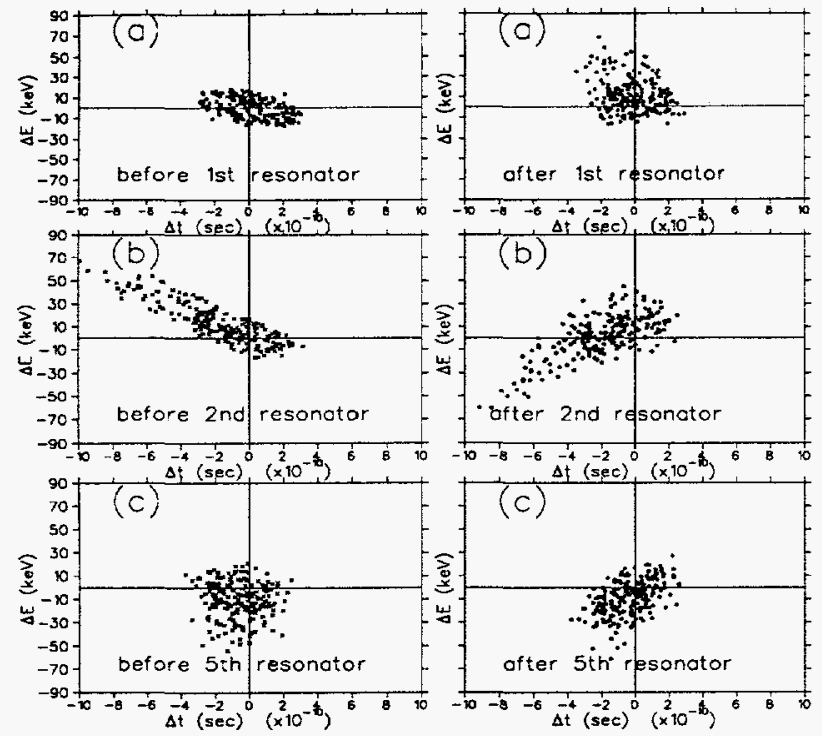

Figure 4: Longitudinal phase spaces at the entrance and the exit of the first, second and fifth resonators shown in the left and right graphs, respectively.

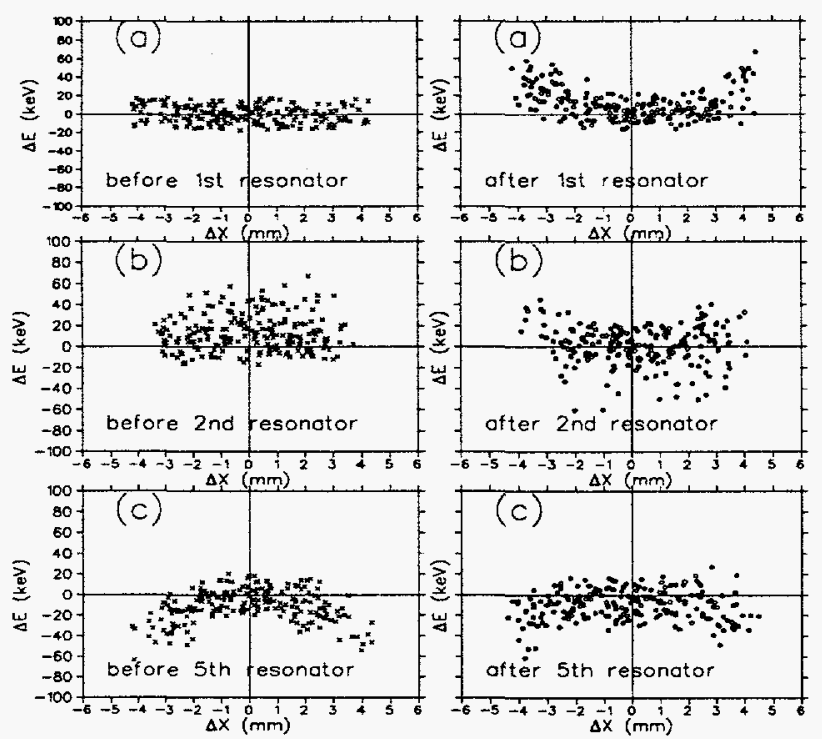

Figure 5: Coupled phase spaces displayed for the corresponding resonators of Figure 4

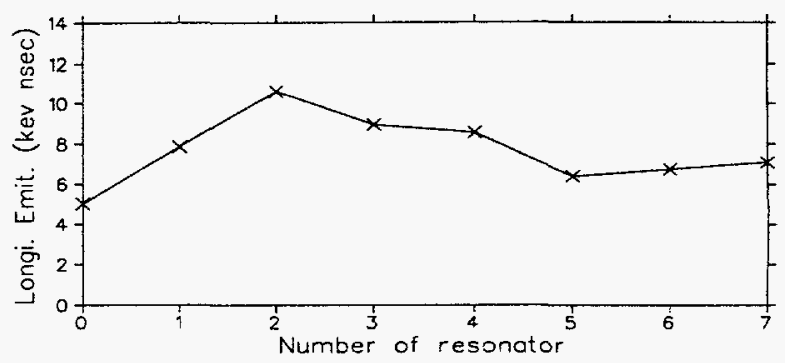

Figure 6: Longitudinal emittance in the first seven resonators of the injector linac.

\section{CONCLUSION}

If the beam size is sufficiently smaller than the wavelength of the slow wave, a beam energy spread due to nonlinear radial variation of energy gain in a drift tube linac can be negligible. However, for low q/A ions at low energies it can be difficult to obtain transverse focusing to insure an adequately small beam. The resulting emittance growth can be corrected using the correlation between transverse and longitudinal phase spaces that we have discussed. This correlation is beneficial for the design of a low energy injector for a radioactive beam facility proposed at ANL, in which high beam quality and large transverse acceptance are essential.

\section{References}

[1] J. Nolen, Accelerator Complex for a Radioactive Ion Beam Facility at ATLAS, in these proceedings.

[2] K. Shepard and J. Kim, A low-charge-state injector linac for ATLAS, in these proceedings.

[3] M. Reiser, Theory and Design of Charged Particle Beams, p142, John Wiley \& Sons, Inc. (1994)

[4] J.W.Kim and K.W.Shepard, High Field Superconducting Quadrupole for a Low Charge State Linac, in these proceedings.

[5] L.M. Bollinger, et.al., Nucl. Instr. and Meth., A328, 221 (1993) 